\title{
A useful protocol for analyses of mutations of the epidermal growth factor receptor gene
}

\author{
DAGE LIU $^{1}$, JUN NAKANO $^{1}$, MASAKI UENO $^{2}$, DAIKI MASUYA $^{1}$, TAKASHI NAKASHIMA $^{1}$, \\ HIROYASU YOKOMISE ${ }^{1}$, KOUICHI YUBE ${ }^{3}$ and CHENG-LONG HUANG ${ }^{1}$ \\ ${ }^{1}$ Second Department of Surgery, ${ }^{2}$ Department of Pathology and Host Defense, ${ }^{3}$ Life Science Research Center, \\ Kagawa University, 1750-1, Miki-cho, Kita-gun, Kagawa 761-0793, Japan \\ Received January 18, 2006; Accepted March 10, 2006
}

\begin{abstract}
Recent studies have reported that mutations of the epidermal growth factor receptor (EGFR) gene are associated with the responsiveness of tyrosine kinase inhibitors (TKIs), which are molecular targets for non-small cell lung cancer (NSCLC). To provide genetic analyses for NSCLC patients, a simple and reliable method using paraffin-embedded materials is needed. The DEXPAT DNA extraction kit was used for DNA extraction from paraffin-embedded materials. DNA was amplified using the nested PCR technique, then analyzed by direct sequencing for EGFR mutations (exons 18 to 21). The phenol/chloroform extraction for DNA was also performed for comparison. When the DEXPAT kit was used, distinct bands were observed in all products after nested PCR assays of paraffin-embedded materials. Distinct sequencing signals were obtained. Results from the sequencing analysis of paraffin-embedded materials and frozen materials were completely concordant. The current study suggests that DNA extraction with the DEXPAT kit followed by nested PCR is a simple and reliable technique for analyzing the EGFR gene status with paraffin-embedded samples.
\end{abstract}

\section{Introduction}

Non-small cell lung cancer (NSCLC) is one of the most common malignancies with a poor prognosis. It is important to determine an effective chemotherapy (made-to-order chemotherapy) to improve the clinical outcome of NSCLC patients. The epidermal growth factor receptor (EGFR) encodes a receptor tyrosine kinase, and is a target molecule of tyrosine kinase inhibitors (TKIs), such as gefitinib and erlotinib. Clinical studies revealed the responsiveness of TKIs to be associated with EGFR mutations $(1,2)$. EGFR mutations

Correspondence to: Dr Cheng-long Huang, Second Department of Surgery, Faculty of Medicine, Kagawa University, 1750-1, Mikicho, Kita-gun, Kagawa 761-0793, Japan

E-mail: chuang@kms.ac.jp

Key words: epidermal growth factor receptor, mutation, paraffinembedded materials, non-small cell lung cancer frequently occur in the kinase domain (exons 18 to 21) (3). Therefore, analysis of the EGFR gene status could help to predict the responsiveness of NSCLC patients to TKIs.

In general, frozen specimens are preferable for genetic analyses because their DNA and RNA remain in a good condition. However, frozen specimens are hard to preserve in general hospitals. As a result, most clinical materials are preserved in paraffin blocks after formalin fixation. During formalin fixation, formaldehyde within the tissues gradually changes to formic acid, which hydrolyzes DNA $(4,5)$. Various studies have been conducted to develop better methods of obtaining high quality DNA from paraffin-embedded materials for genetic analyses $(6,7)$. Here, we developed a protocol for analyzing the EGFR gene status using paraffin-embedded samples. Our findings demonstrate the combination of DNA extraction using both the DEXPAT DNA extraction kit (Takara, Otsu, Japan) and nested PCR to be a simple and quick method for analyzing the EGFR gene status using paraffin-embedded materials.

\section{Materials and methods}

Samples. Paraffin-embedded samples and frozen specimens of primary tumors were obtained from 12 NSCLC patients. For DNA extraction from paraffin-embedded samples, a 7- $\mu \mathrm{m}$ thick section was cut from a paraffin block. Normal tissue surrounding the tumor was removed in referencing to H\&Estained slides. When the area of the tumor tissue was less than $6 \times 6 \mathrm{~mm}^{2}$, the number of sections was increased 2- or 3fold according to its area.

DNA extraction. We used the DEXPAT DNA extraction kit (Takara) to perform DNA extraction from paraffin-embedded samples and frozen specimens. A tumor sample and $500 \mu 1$ of well-mixed DEXPAT solution were placed in a $1.5-\mathrm{ml}$ tube, which was boiled at $100^{\circ} \mathrm{C}$ for $10 \mathrm{~min}$. After centrifuging at $12,000 \mathrm{rpm}$ for $10 \mathrm{~min}$ at $4^{\circ} \mathrm{C}$, the supernatant was used as a PCR template. In total, this procedure required only $25 \mathrm{~min}$ to complete. For the control groups, phenol/chloroform extraction was also performed for DNA extraction of paraffinembedded samples and frozen specimens.

Nested PCR. We used three sets of primers for each exon (exons 18 to 21 ) of the EGFR gene, including first PCR 
Table I. PCR and sequencing primers for the epidermal growth factor receptor gene.

\begin{tabular}{|c|c|c|c|c|}
\hline Exon & $\begin{array}{l}\text { Exon size } \\
\quad(\mathrm{bp})\end{array}$ & Forward primer & Reverse primer & $\begin{array}{l}\text { Product } \\
\text { size (bp) }\end{array}$ \\
\hline \multirow[t]{3}{*}{ Exon 18} & 122 & $18-1 \mathrm{~F}$, caa atg agc tgg caa gtg ccg tgt c & $18-1 \mathrm{R}$, gag ttt ccc aaa cac tca gtg aaa c & 400 \\
\hline & & $18-2 \mathrm{~F}$, caa gtg ccg tgt cct ggc acc caa gc & $18-2 \mathrm{R}$, cca aac act cag tga aac aaa gag & 381 \\
\hline & & 18-SF, ctg gca ctg ctt tcc agc & 18-SR, aca gct tgc aag gac tct gg & 344 \\
\hline \multirow[t]{3}{*}{ Exon 19} & 99 & $19-1 F$, gca ata tca gcc tta ggt gcg gtc & 19-1R, cat aga aag tga aca ttt agg atg tg & 372 \\
\hline & & $19-2 \mathrm{~F}$, cct tag gtg cgg ctc cac agc & $19-2 R$, cat tta gga tgt gga gat gag $c$ & 349 \\
\hline & & 19-SF, tcg ctg gta aca tcc acc cag atc ac & 19-SR, ctc tga acc tca ggc cca cct ttt ct & 248 \\
\hline \multirow[t]{3}{*}{ Exon 20} & 186 & $20-1 \mathrm{~F}$, cca tga gta cgt att ttg aaa ctc & $20-1 \mathrm{R}$, cat atc ccc atg gca aac tct tgc & 408 \\
\hline & & $20-2 \mathrm{~F}$, gaa act caa gat cgc att cat gc & $20-2 R$, gca aac tct tgc tat ccc agg ag & 379 \\
\hline & & 20-SF, tct tca cct gga agg ggt c & 20-SR, cta tcc cag gag cgc aga c & 273 \\
\hline \multirow[t]{3}{*}{ Exon 21} & 156 & $21-1 F$, cta acg ttc gcc agc cat aag tcc & $20-1 \mathrm{R}$, gct gcg agc tca ccc aga atg tct gg & 415 \\
\hline & & $21-2 \mathrm{~F}$, cag cca taa gtc ctc gac gtg g & 20-2R, cat cct ccc ctg cat gtg tta aac & 374 \\
\hline & & 21-SF, gaa ttc gga tgc aga gct t & 20-SR, agg tca gec agc att ttc & 256 \\
\hline
\end{tabular}

F, forward; R, reverse; 1, first round primer; 2, second round primer; S, Sequencing.

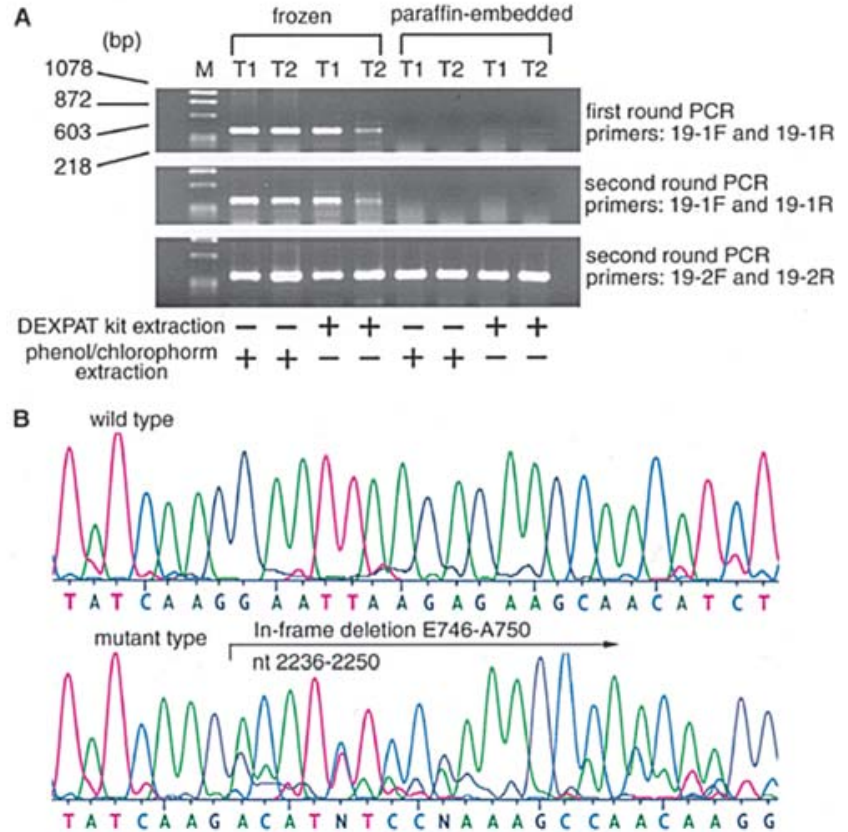

Figure 1. (A) Agarose gel electrophoresis of PCR products (exon 19) using DNA samples extracted by the DEXPAT kit or phenol/chloroform from two NSCLC tumors. (B) Nucleotide sequence of the EGFR gene with in-frame deletions within the tyrosine kinase domain from a paraffin-embedded specimen.

primers, second PCR primers, and sequence primers (Table I) (2). The first round PCR amplification was performed in a $20-\mu 1$ reaction mixture containing $0.5 \mu 1$ of Taq polymerase (Takara), $2 \mu 1$ of $10 \mathrm{X}$ buffer, $2 \mu 1$ of dNTP mixture, $0.5 \mu 1$ of $30 \mu \mathrm{M}$ of a first forward primer and a first reverse primer, and $2 \mu \mathrm{l}$ of supernatant after DEXPAT extraction. The reaction mixture was subjected to $41 \mathrm{PCR}$ amplification cycles of $30 \mathrm{sec}$ at $94^{\circ} \mathrm{C}, 30 \mathrm{sec}$ at $60^{\circ} \mathrm{C}$, and $45 \mathrm{sec}$ at $72^{\circ} \mathrm{C}$. The $2 \mu 1$ of the first round PCR product was then applied to the second round PCR with second PCR primers using the same protocols used in the first round PCR. Each PCR product was confirmed by electrophoresis in $1 \%$ agarose gel containing ethidium bromide.

Sequencing analysis. The PCR product was directly subjected to sequencing analysis. Sequencing reactions were performed using a BigDye Terminator cycle sequencing kit (Applied Biosystems, Foster City, CA, USA) according to the manufacturer's protocol and then analyzed on an ABI PRISM DNA sequencer (Applied Biosystems).

\section{Results and discussion}

Regarding the paraffin-embedded samples, we evaluated the PCR products using samples with DEXPAT extraction or phenol/chloroform extraction. When phenol/chloroform extraction was used for DNA extraction of paraffin-embedded samples, there was no visible band in the first round PCR products from all samples, while distinct bands were observed in $83.3 \%$ (44 of 48 ) of second round PCR products after nested PCR (Fig. 1A). In contrast, when DEXPAT extraction was used, no band was seen in any first round PCR products, and distinct bands were observed in all second round PCR products after nested PCR. Regarding DNA extraction from paraffin-embedded samples, DEXPAT extraction is significantly better than conventional phenol/chloroform extraction ( $\mathrm{P}=0.0034)$.

Regarding frozen specimens, after first round PCR, distinct bands were observed in all PCR products using phenol/chloroform extraction, and $60 \%$ of PCR products using DEXPAT extraction (Fig. 1A). Even when DEXPAT extraction was used, clear bands were intensely observed in all PCR products after nested PCR assays.

The DEXPAT kit extracts the DNA template from tissue samples using a boiling method and fibers that remove PCR inhibitors. DNA fragments in each exon of the EGFR gene 
(exons 18 to 21) range from 99 to $186 \mathrm{bp}$. We designed the first PCR primers so the PCR products have sufficient length to keep base pairs in the intron to later perform accurate sequence analyses. Consequently, products from the first PCR assays using first primers range from 372 to $415 \mathrm{bp}$ (Table I). The DEXPAT kit is reported to extract less than $400 \mathrm{bp}$ of the DNA fragment. Although the boiling method is not appropriate for the extraction of long DNA fragments, DNA is degenerated into small fragments in paraffin-embedded samples, as previously mentioned. The present study demonstrated that the DEXPAT kit is a useful method for analyzing the EGFR gene using paraffin-embedded samples.

Furthermore, DNA extraction with the DEXPAT kit is simple to perform and requires only $25 \mathrm{~min}$ to complete. Finally, $250 \mu 1$ of supernatant can be obtained for further PCR assays. This method is also appropriate for evaluating other genes associated with tumor biology, such as the p53 or $\mathrm{K}$-ras gene. Therefore, DEXPAT extraction is considered to be clinically useful not only for paraffin-embedded samples, but also for frozen specimens.

Nested PCR was originally developed to rule out nonspecific products during PCR amplification. The second PCR primers, designed in the internal region of products of first round PCR assays, were considered to play a critically important role in PCR amplification, especially for degenerated DNA fragments in paraffin-embedded samples. In fact, the present study has demonstrated that nested PCR is effective and essential for PCR amplification with DNA from paraffinembedded samples, extracted not only with the DEXPAT kit but also with phenol/chloroform. In contrast, there were only faint bands observed in second round PCR products when the first PCR primers were used for second round PCR assays (Fig. 1A). As a result, nested PCR is considered necessary for the analysis of EGFR gene status when using paraffinembedded samples.

Furthermore, we also designed sequence primers located in the internal region of the products from nested PCR assays. We considered that the designation of primers, including the second PCR primers and sequence primers, is important for a sufficient DNA template to contribute to credible sequencing signals. Consequently, distinct sequencing signals were obtained in all samples using not only frozen specimens, but also paraffin-embedded samples (Fig. 1B). The results of sequencing analyses obtained from paraffin-embedded materials and frozen materials were concordant.

In conclusion, we demonstrated the combination of DNA extraction with the DEXPAT kit and nested PCR to be clinically useful for DNA analyses of formalin-fixed, paraffinembedded materials. This protocol is easy and reliable, supplying a sufficient template for credible sequencing analysis of EGFR gene status. The use of this new method should allow us to clinically predict the responsiveness of NSCLC patients to TKIs, and provide more clinical options for these patients.

\section{References}

1. Paez JG, Janne PA, Lee JC, Tracy S, Greulich H, Gabriel S, Herman P, Kaye FJ, Lindeman N, Boggon TJ, Naoki K, Sasaki H, Fujii Y, Eck MJ, Sellers WR, Johnson BE and Meyerson M: EGFR mutations in lung cancer: correlation with clinical response to gefitinib therapy. Science 304: 1497-1500, 2004

2. Pao W, Miller V, Zakowski M, Doherty J, Politi K, Sarkaria I, Singh B, Heelan R, Rusch V, Fulton L, Mardis E, Kupfer D, Wilson R, Kris M and Varmus H: EGF receptor gene mutations are common in lung cancers from 'never smokers' and are associated with sensitivity of tumors to gefitinib and erlotinib. Proc Natl Acad Sci USA 101: 13306-13311, 2004.

3. Kosaka T, Yatabe Y, Endoh H, Kuwano H, Takahashi T and Mitsudomi T: Mutations of the epidermal growth factor receptor gene in lung cancer: biological and clinical implications. Cancer Res 64: 8919-8923, 2004

4. Greer CE, Wheeler CM and Manos MM: Sample preparation and PCR amplification from paraffin-embedded tissues. PCR Methods Appl 3: S113-S122, 1994.

5. Pavelic J, Gall-Troselj K, Bosnar MH, Kardum MM and Pavelic K: PCR amplification of DNA from archival specimens. A methodological approach. Neoplasma 43: 75-81, 1996.

6. Sato Y, Sugie R, Tsuchiya B, Kameya T, Natori M and Mukai K: Comparison of the DNA extraction methods for polymerase chain reaction amplification from formalin-fixed and paraffinembedded tissues. Diagn Mol Pathol 10: 265-271, 2001.

7. Shi SR, Cote RJ, Wu L, Liu C, Datar R, Shi Y, Liu D, Lim H and Taylor CR: DNA extraction from archival formalin-fixed, paraffin-embedded tissue sections based on the antigen retrieval principle: heating under the influence of $\mathrm{pH}$. J Histochem Cytochem 50: 1005-1011, 2002. 